\title{
A Phase II Study of Tri-weekly Low-dose Nab-paclitaxel Chemotherapy for Patients with Advanced Gastric Cancer
}

\author{
SHO SATO ${ }^{1}$, CHIKARA KUNISAKI $^{1}$, YUSAKU TANAKA ${ }^{1}$, KEI SATO ${ }^{1}$, \\ HIROSHI MIYAMOTO ${ }^{1}$, NORIO YUKAWA ${ }^{1}$, YOSHIRO FUJII ${ }^{1}$, JUN KIMURA ${ }^{2}$, \\ RYO TAKAGAWA ${ }^{3}$, MASAZUMI TAKAHASHI ${ }^{4}$, TAKASHI KOSAKA ${ }^{5}$, HIROTOSHI AKIYAMA ${ }^{5}$, \\ YUSUKE SAIGUSA ${ }^{6}$, MASATAKA TAGURI $^{6}$, TAKEHARU YAMANAKA $^{6}$ and ITARU ENDO ${ }^{5}$ \\ ${ }^{1}$ Department of Surgery, Gastroenterological Center, Yokohama City University, Yokohama, Japan; \\ ${ }^{2}$ Department of Surgery, Yokosuka Kyousai Hospital, Yokosuka, Japan; \\ ${ }^{3}$ Department of Surgery, Saiseikai Yokohamashi Nanbu Hospital, Yokohama, Japan; \\ ${ }^{4}$ Department of Gastroenterological Surgery, Yokohama Municipal Citizen's Hospital, Yokohama, Japan; \\ ${ }^{5}$ Department of Gastroenterological Surgery, \\ Yokohama City University Graduate School of Medicine, Yokohama, Japan; \\ ${ }^{6}$ Department of Clinical Statistics, Yokohama City University Graduate School of Medicine, Yokohama, Japan
}

\begin{abstract}
Background/Aim: Nanoparticle albumin-bound (nab)-paclitaxel has demonstrated antitumor activity against advanced gastric cancer. However, gastric cancer patients can be difficult to treat with the recommended dose because of the high incidence of adverse toxicities. The aim of this study was to evaluate the safety and effectiveness of low-dose nabpaclitaxel in a multicenter, single-arm, phase II study. Patients and Methods: Treatment included low doses of $180 \mathrm{mg} / \mathrm{m}^{2} \mathrm{nab}$ paclitaxel administered on day 1 of each 21-day cycle. The primary endpoint was defined as the overall response rate (ORR). The secondary endpoints included progression-free survival (PFS), safety, and overall survival (OS). A total of 34 patients were enrolled in the full-analysis set. Results: The ORR was 5.9\%. The median PFS and OS were 2.4 months and 9.2 months, respectively. The most common grade 3/4 toxicities were anemia (8.8\%), neutropenia (5.9\%), appetite loss (5.9\%) and peripheral sensory neuropathy (5.9\%). No treatmentrelated deaths occurred. Conclusion: The tri-weekly low dose of nab-paclitaxel therapy is effective towards advanced gastric cancer patients with good tolerability and an acceptable margin of safety.
\end{abstract}

Gastric cancer is the third leading cause of cancer-related death worldwide, accounting for 723,000 deaths yearly, or

Correspondence to: Chikara Kunisaki, MD, Ph.D., 4-57 Urafunecho, Minami-ku, Yokohama, 232-0024, Japan. Tel: +81 452615656, Fax: +81 452619492, e-mail: s0714@med.yokohama-cu.ac.jp

Key Words: Nab-paclitaxel, gastric cancer, chemotherapy, triweekly.
$8.8 \%$ of the annual deaths from cancer $(1,2)$. Platinum drugs plus fluoropyrimidine combination therapy is widely used as first-line chemotherapy for unresectable gastric cancer (3), and the survival benefit of second-line chemotherapy using docetaxel or irinotecan has recently been confirmed in several randomized trials (4-6). Nab-paclitaxel is a nanoparticle albumin-bound (nab), solvent-free formulation of paclitaxel. Solvent-based paclitaxel can cause hypersensitivity and anaphylactic reactions in certain patients, which are mainly related to the polyethoxylated castor oil vehicle $(7,8)$. Nab-paclitaxel is free of polyethoxylated castor oil, minimizing the risk of hypersensitivity reactions without pre-medications $(9,10)$. Additionally, because this formation does not require hydrated ethanol as a solvent, it can be used in patients with alcohol intolerance. Furthermore, higher doses of nabpaclitaxel can be administered over a shorter infusion time compared to solvent-based paclitaxel. At equal doses, paclitaxel dose was $33 \%$ higher for nab-paclitaxel versus solvent-based paclitaxel, indicating more effective intratumoral accumulation of nab-paclitaxel $(11,12)$. A phase II study of triweekly nab-paclitaxel administration at 260 $\mathrm{mg} / \mathrm{m}^{2}$ for patients with advanced gastric cancer who suffered from progression despite having received fluoropyrimidine-containing treatment, yielded relatively good responses, with an overall response rate of $27.8 \%$, disease control rate of $59.3 \%$, progression-free survival of 2.9 months, and overall survival of 9.2 months. However, grade $3 / 4$ toxicities, including neutropenia $(49.1 \%)$ and peripheral sensory neuropathy $(23.6 \%)$ occurred at a high frequency (13), making it difficult to treat patients with the recommended dose (14). 
A phase II study of low-dose nab-paclitaxel $\left(180 \mathrm{mg} / \mathrm{m}^{2}\right)$ in patients with metastatic or recurrent breast cancer showed no grade $3 / 4$ peripheral sensory neuropathy with a relatively good response rate (15). The aim of chemotherapy against advanced gastric cancer is to improve overall survival, suppress clinical symptoms due to cancer progression, and maintain quality of life (QOL). In this regard, it is necessary to develop a less toxic chemotherapy regimen in order to properly treat patients with advanced gastric cancer. This phase II study aimed to investigate the potential benefits of low-dose $\left(180 \mathrm{mg} / \mathrm{m}^{2}\right)$ nab-paclitaxel treatment as the secondline regimen for patients with advanced gastric cancer.

\section{Patients and Methods}

Study objectives and design. The present study was a nonrandomized, open-label, multicenter phase II registration trial of patients with unresectable or recurrent gastric cancer who had failed to obtain favorable outcomes after previous treatment with a fluoropyrimidine-based regimen. This study was conducted in accordance with the Declaration of Helsinki and was approved by the review board of each institution. All participants provided written informed consent prior to study entry. The study protocol was registered at the University Hospital Information Network (UMIN) Clinical Trials Registry (protocol ID UMIN000016973).

Eligibility criteria. All eligible patients had to have histologically confirmed metastatic or recurrent gastric cancer, be between 20 and 80 years of age, an Eastern Cooperative Oncology Group performance status (PS) 0-2, had at least one prior chemotherapy with fluoropyrimidine-containing drug, no metastasis in the central nervous system, adequate bone marrow function (absolute neutrophil count $\geq 1,500$ cells $/ \mathrm{mm}^{2}$, platelet count $>100,000$ cells $/ \mathrm{mm}^{2}$ ), adequate liver function (total bilirubin $\leq 1.5 \mathrm{mg} / \mathrm{dl}$ and serum transaminase $\leq 100 \mathrm{IU} / \mathrm{l}$ ), adequate renal function (serum creatinine $\leq 1.5 \mathrm{mg} / \mathrm{dl}$ ), and a life expectancy $\geq 90$ days. Patients were excluded if they had other severe medical conditions, including other active malignancies, or if they had a history of other nab-paclitaxel treatments.

Treatment schedule and evaluation of toxicity. Nab-paclitaxel (Abraxane; Taiho Pharmaceutical Company, Tokyo, Japan) was administered at a dose of $180 \mathrm{mg} / \mathrm{m} 2$ over $30 \mathrm{~min}$ by intravenous infusion on day 1 of a 3 -week cycle. This treatment was repeated every 3 weeks until disease progression or unacceptable toxicity was observed. Toxicity was graded according to the Common Terminology Criteria for Adverse Events (16). Nab-paclitaxel could be administered if neutrophil count was $\geq 1,500$ cells $/ \mathrm{m}^{3}$, platelet count was $\geq 100,000 / \mathrm{m}^{3}$ and all relevant non-hematological toxicities were grade 2 or lower. Treatment dose reductions were planned for neutropenia grade 4, thrombocytopenia grade 3 or nonhematological grade 3 toxicities, in which nab-paclitaxel was to be re-introduced at a dose of $140 \mathrm{mg} / \mathrm{m} 2$. Treatment could be withheld for up to 40 days. If either non-hematological grade 4 toxicities, same-grade adverse events after dose reduction, or allergic grade 3 toxicities were observed, patients were excluded from the study.

Endpoints and evaluation of treatment. The primary endpoint was overall response rate (ORR). Tumor response was evaluated every
6-9 weeks by computed tomography. Measurable lesions were assessed according to RECIST version 1.1 and were classified as complete response (CR), partial response (PR), stable disease (SD), or progressive disease (PD). Response rate was defined as the sum of $\mathrm{CR}$ rate and $\mathrm{PR}$ rate (17). Secondary endpoints included progression-free survival (PFS), overall survival (OS), and safety. PFS was defined as the time from the date of randomization in the clinical trial until disease progression or death from any cause. A full-analysis set (FAS) was used to evaluate patients.

Statistical analysis. In the phase II J-0200 trial, the response rate of full-dose nab-paclitaxel was $27.8 \%$ (13). It was hypothesized that the regimen would be effective if the response rate reached $25 \%$. Under this assumption, the estimated required sample size would be 44 cases; in this trial, a total of 45 patients were observed, with the aim of recruiting more to allow for follow-up loss. The recruiting period was determined as 3 years from the first patient enrollment which was November 2015, and the end of the observation period was determined as a year after last patient enrollment. However, the outcomes of a phase III trial using ramucirumab were disclosed during this study (18), and so it was difficult to recruit further patients in spite of the elongation of the patient enrollment period. Subsequently, a total of 37 patients were enrolled in this study. The clinical cut-off date for this study was April 2018. PFS and OS were estimated using the Kaplan-Meier method. Data were analyzed statistically using SAS Version 9.4 (SAS Institute Inc., NC, USA).

\section{Results}

Patient characteristics. Between November 2015 and April 2017, 37 patients were assigned to receive low-dose nabpaclitaxel every 3 weeks. Three patients who did not meet the eligibility criteria were excluded from the full analysis set. Median follow-up for overall survival was 8.3 months, and 26 patients had died by the end of the study. The most common cause of discontinuation was progressive disease. Two patients underwent surgery during the trial, one for successful conversion surgery and one for exploratory laparotomy (Figure 1).

The major clinicopathological characteristics of the patients are listed in Table I. S-1 fluoropyrdimidine was most frequently used as the first-line chemotherapy regimen among fluoropyrimidine-based regimens (79.4\%). Monotherapy was performed in five patients $(14.7 \%)$, doublet chemotherapy in 24 patients $(70.6 \%)$, and triplet chemotherapy in five patients (14.7\%) (Table II). Post chemotherapy treatments are shown in Table II. Chemotherapy was performed in $55.9 \%$ of patients and conversion surgery was performed in $2.9 \%$. Best supportive care was performed in $44.1 \%$ of patients. Chemotherapy regimens included irinotecan-based regimen in $32.4 \%$ of patients, ramucirumab in $5.9 \%$, nab-paclitaxel after drug holiday ( $>40$ days) in $5.9 \%$ and other regimens in $5.9 \%$.

Anti-tumor activity. RECIST response was assessable in 34 patients. The overall response rate (ORR) was $5.9 \%$ (2), 


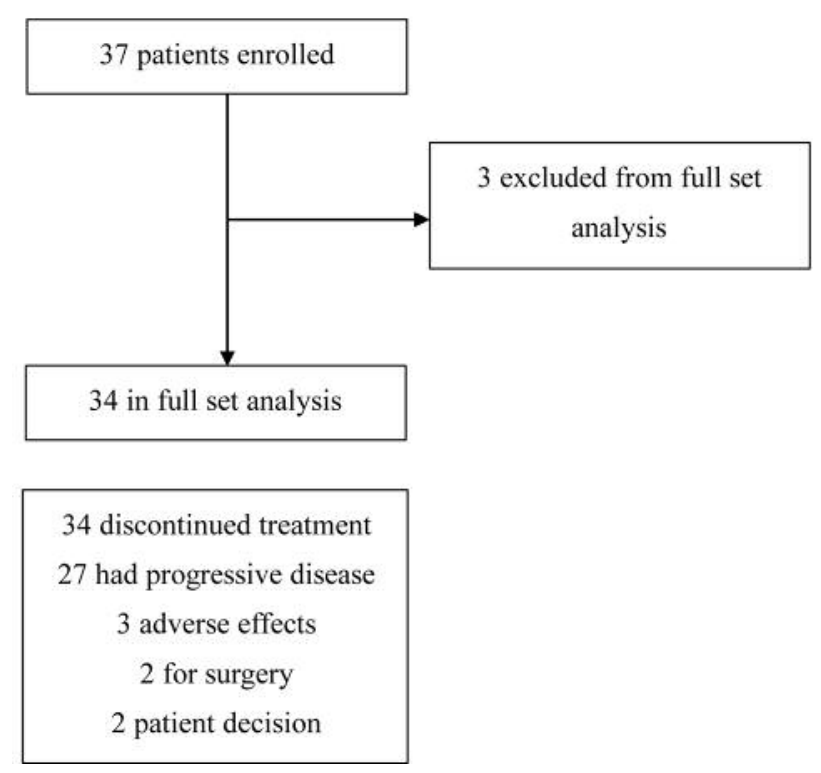

Figure 1. Patient flow diagram.

including $\mathrm{CR}$ in one patient and PR in another. Fourteen patients showed SD, and disease progressed in 18 patients. The disease control rate (DCR) $(\mathrm{CR}+\mathrm{PR}+\mathrm{SD})$ was $47.1 \%$ (Table III). The median OS was 9.2 months $(95 \% \mathrm{CI}=6.4$ 19.1) and median PFS was 2.4 months $(95 \% \mathrm{CI}=1.8-4.6)$ (Figure 2).

Toxicity. The median number of the treatment cycles was three (range $=1-19$ cycles). All patients were evaluable for toxicity (Table IV). No death due to toxicity was observed. Hematological toxicity was mainly represented by anemia recorded in 26 patients $(76.4 \%)$, with grade 3-4 anemia recorded in $8.8 \%$. Neutropenia was observed in 14 patients $(41.2 \%)$, with grade 3-4 neutropenia recorded in two (5.9\%). Non-hematological toxicity was mainly represented by peripheral sensory toxicity $(61.8 \%)$ and alopecia $(58.8 \%)$. Grade 3 peripheral sensory toxicity was observed in two patients (5.9\%). Two patients (5.9\%) experienced grade 3 appetite loss and one patient $(2.9 \%)$ experienced grade 3 nausea. Median relative dose intensity (\%) was 100 (range $=68.9-100)$. Dose reduction of nab-paclitaxel was required in one patient $(2.9 \%)$ patient due to grade 4 thrombocytopenia. All patients received chemotherapy in an out-patient clinic.

\section{Discussion}

Our results demonstrate that low doses of nab-paclitaxel $\left(180 \mathrm{mg} / \mathrm{m}^{2} \mathrm{q} 3 \mathrm{~W}\right)$ chemotherapy as second-line treatment, administered once every 3 weeks, are oncologically
Table I. Patient characteristics.

\begin{tabular}{lc}
\hline Variables & $\mathrm{n}=34$ \\
\hline Gender, $\mathrm{n}(\%)$ & \\
Male & $25(73.53)$ \\
Female & $9(26.47)$ \\
Age & \\
Median (Range) & $68(51-80)$ \\
Mean (SD) & $67.8(8.0)$ \\
PS*, n (\%) & \\
0 & $28(82.35)$ \\
1 & $6(17.65)$ \\
BMI & \\
Median (Range) & $20.17(14.5-26.3)$ \\
Mean (SD) & $20.07(3.22)$ \\
BSA & \\
Median (Range) & $1.545(1.11-1.87)$ \\
Mean (SD) & $1.538(0.164)$ \\
Type of treatment, $\mathrm{n}(\%)$ & \\
Recurrence after gastrectomy & $6(17.65)$ \\
Progressive disease during first-line chemotherapy & $28(82.35)$ \\
Macroscopic type, $\mathrm{n}(\%)$ & \\
Unknown & $1(2.94)$ \\
1 & $0(0)$ \\
2 & $10(29.41)$ \\
3 & $14(41.18)$ \\
4 & $7(20.59)$ \\
5 & $2(5.88)$ \\
Histological type & \\
Pap & $2(5.88)$ \\
Tub1 & $5(23.53)$ \\
Tub2 & $5(14.71)$ \\
Por1 & $2(5.88)$ \\
Por2 & $2(5.88)$ \\
Sig & \\
Muc & \\
HER2, n (\%) & \\
Positive & \\
Negative & \\
\hline & \\
\hline &
\end{tabular}

PS: Performance status.

effective with good tolerability for patients with advanced gastric cancer. The median OS was 9.2 months and median PFS was 2.4 months. Although the majority of adverse events were mild, two patients suffered from grade 3 peripheral sensory neuropathy.

Previously, a phase II clinical trial of nab-paclitaxel $\left(260 \mathrm{mg} / \mathrm{m}^{2} \mathrm{q} 3 \mathrm{~W}\right)$ conducted in Japan showed promising activity against previously treated advanced gastric cancers (13), where the ORR was $27.8 \%$ and the DCR was $59.3 \%$, with median PFS of 2.9 months and median OS of 9.2 months. In the current study, PFS and OS were equivalent, though ORR and DCR were inferior. The previous study used relative dose intensity (RDI) of $93.4 \% \quad(80.9$ $\mathrm{mg} / \mathrm{m}^{2} /$ week), $20.9 \mathrm{mg} / \mathrm{m}^{2}$ higher than the current study (RDI: $60 \mathrm{mg} / \mathrm{m}^{2} /$ week), indicating that the lower dose of 
Table II. Previous and post-chemotherapy regimen.

\begin{tabular}{lc}
\hline Previous chemotherapy regimen & $\mathrm{n}(\%)$ \\
\hline Monotherapy & \\
S-1 & $5(14.71)$ \\
Doublet chemotherapy & $11(32.35)$ \\
S-1+Cisplatin & $5(14.71)$ \\
S-1+Oxaliplatin & $5(14.71)$ \\
Capecitabine+Cisplatin & $2(5.88)$ \\
S-1+Docetaxel & $1(2.94)$ \\
Capecitabine+Cisplatin $\rightarrow$ S-1 & \\
Triplet chemotherapy & $3(8.82)$ \\
S-1+Docetaxel+Cisplatin & $2(5.88)$ \\
Capecitabine+Cisplatin+Trastuzumab & $\mathrm{n}(\%)$ \\
\hline Post chemotherapy treatment & \\
\hline Chemotherapy & $7(20.59)$ \\
Irinotecan & $4(11.76)$ \\
Irinotecan+Cisplatin & $2(5.88)$ \\
Ramucirumab & $2(5.88)$ \\
Nab-paclitaxel (after drug holiday) & $1(2.94)$ \\
Capecitabine+Cisplatin & $1(2.94)$ \\
Docetaxel+Cisplatin & $1(2.94)$ \\
Surgery & $1(2.94)$ \\
Conversion surgery $\rightarrow$ nab-paclitaxel & $15(44.12)$ \\
Exploratory laparotomy & \\
Best supportive care & \\
\hline
\end{tabular}

Table III. Anti-tumor activity.

\begin{tabular}{lc}
\hline & $\mathrm{n}(\%)$ \\
\hline Best response & \\
Complete response & $1(2.9)$ \\
Partial response & $1(2.9)$ \\
Stable disease & $14(41.2)$ \\
Progressive disease & $18(52.9)$ \\
Overall response & $2(5.9)$ \\
\hline
\end{tabular}

nab-paclitaxel is still effective. Appropriate RDI of nabpaclitaxel to achieve survival benefit maintaining quality of life (QOL) may be lower than previously suggested. Under the higher-dose regimen, tri-weekly administration led to high frequency of severe adverse effects, including grade 3 peripheral sensory neuropathy and grade $3 / 4$ neutropenia notably in $23.6 \%$ and $49.1 \%$ of patients, respectively (13). In contrast, the current study showed that grade 3 peripheral sensory neuropathy and grade $3 / 4$ neutropenia occurred in only $2.9 \%$ and $5.9 \%$ of patients, and only one patient required dose reduction due to an adverse effect. Based on these outcomes, we attribute the mild adverse toxicity to the low dosage of nab-paclitaxel. Major adverse reactions
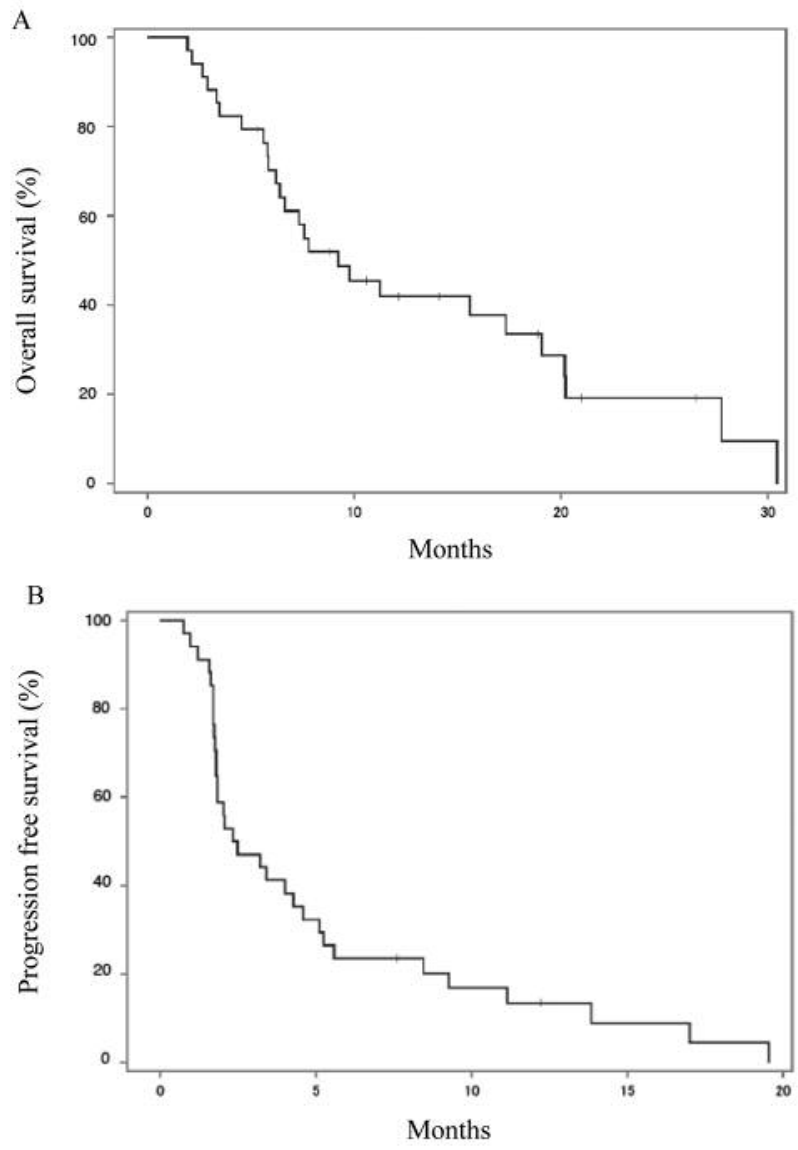

Figure 2. Kaplan-Meier plots of overall survival (A) and progressionfree survival $(B)$ in the full analysis set of patients with advanced gastric cancer receiving low-dose nab-paclitaxel. Median OS: 9.2 months (95\% CI=6.4-19.1), median PFS: 2.4 months (95\%CI=1.8-4.6).

related to myelosuppression and peripheral sensory neuropathy are primary causes of chemotherapy discontinuation. Therefore, an initial reduction in the dose of nab-paclitaxel may be a preferable treatment option to prevent severe adverse events and continue chemotherapy, particularly in an outpatient clinic. Although the dose of nab-paclitaxel in the current study was determined by referring to a previous phase II study for breast cancer (15), it is imperative to consider the effectiveness and safety of another low dose of triweekly nab-paclitaxel regimen (e.g.: $80 \%$ of the regular dose) in a future study.

Recently, a phase III trial showed that weekly nabpaclitaxel was non-inferior to weekly solvent-based paclitaxel, whereas nab-paclitaxel every 3 weeks was not non-inferior to solvent-based paclitaxel (ABSOLUTE trial) (3). The median treatment duration was 3.7 months and median OS was 11.1 months in the weekly nab-paclitaxel group, which showed more favorable survival durations 
Table IV. Adverse drug reactions. $n$ (\%).

\begin{tabular}{lcll}
\hline & Grade $1-2$ & Grade 3 & Grade 4 \\
\hline Hematological toxicity & & & \\
Anemia & $23(67.6)$ & $2(5.9)$ & $1(2.9)$ \\
Leucopenia & $12(35.3)$ & $2(5.9)$ & $0(0)$ \\
Neutropenia & $12(35.3)$ & $1(2.9)$ & $1(2.9)$ \\
Thrombocytopenia & $6(17.7)$ & $0(0)$ & $1(0)$ \\
Febrile neutropenia & $0(0)$ & $0(0)$ & $0(0)$ \\
Non-hematologic toxicity & & & \\
Alopecia & $20(58.8)$ & $0(0)$ & $0(0)$ \\
Peripheral sensory neuropathy & $19(55.9)$ & $2(5.9)$ & $0(0)$ \\
Fatigue & $17(50.0)$ & $0(0)$ & $0(0)$ \\
AST elevation & $10(29.4)$ & $1(2.9)$ & $0(0)$ \\
Appetite loss & $10(29.4)$ & $2(5.9)$ & $0(0)$ \\
Creatinine elevation & $9(26.5)$ & $1(2.9)$ & $0(0)$ \\
ALT elevation & $8(23.5)$ & $1(2.9)$ & $0(0)$ \\
Nausea & $6(17.6)$ & $1(2.9)$ & $0(0)$ \\
Rash & $6(17.6)$ & $0(0)$ & $0(0)$ \\
Peripheral motor neuropathy & $3(8.8)$ & $1(2.9)$ & $0(0)$ \\
Pyrexia & $3(8.8)$ & $1(2.9)$ & $0(0)$ \\
Diarrhea & $2(5.9)$ & $1(0)$ & $0(0)$ \\
Constipation & $2(5.9)$ & $0(0)$ & $0(0)$ \\
Vomiting & $2(5.9)$ & $0(0)$ & $0(0)$ \\
Arthralgia & $2(5.9)$ & $0(0)$ & $0(0)$ \\
Hyperbilirubinemia & $2(5.9)$ & $0(0)$ & $0(0)$ \\
Mucositis & $1(2.9)$ & $0(0)$ & $0(0)$ \\
Myalgia & $0(0)$ & $0(0)$ & $0(0)$ \\
\hline & & & \\
\hline & & &
\end{tabular}

than in our study. Although neutropenia was highly observed $(39 \%)$ in the weekly nab-paclitaxel group, the weekly nab-paclitaxel treatment may have a higher antitumor activity than our low-dose triweekly nab-paclitaxel group. In this phase III study, RDI of the tri-weekly nabpaclitaxel group and the weekly nab-paclitaxel group were $76.3 \mathrm{mg} / \mathrm{m}^{2} /$ week and $62.9 \mathrm{mg} / \mathrm{m}^{2} /$ week, respectively. A greater amount of the tri-weekly nab-paclitaxel administration might lead to higher incidence of adverse toxicities and as a result, the continuation of treatment will be shortened and this regimen will fail to show a noninferior outcome. This result suggests that the initial dose reduction is an appropriate option for the tri-weekly regimen in order to enable its continuation. In addition, the incidence of PD during first-line chemotherapy was higher in the triweekly nab-paclitaxel group than the other two groups (3). This suggests that malignant potential and tumor volume might be higher in the triweekly nabpaclitaxel group than the other two groups.

In another phase III study, ramucirumab, an antiVEGFR2 antibody, plus solvent-based paclitaxel significantly improved overall survival compared to solvent-based paclitaxel alone as a second-line chemotherapy (RAINBOW trial) (18). Thus, ramucirumab plus paclitaxel therapy is regarded as a standard second-line treatment for advanced gastric cancer in Japan (19). However, this regimen cannot be applied in a subset of the population. First, patients with alcohol intolerance or with hypersensitivity to polyethoxylated castor oil cannot receive solvent-based paclitaxel (20). Nab-paclitaxel is devoid of any solvent or ethanol, thus reducing the risk of hypersensitivity and avoiding the requirement for steroid and antihistamine premedication (13). Hypersensitivity was not observed in our study. Secondly, ramucirumab is considered to be high risk for patients with a thromboembolic disorder (21), hypertension, or deeply ulcerated tumors suspected of gastrointestinal perforation or bleeding. For these patients, low-dose triweekly nabpaclitaxel may be a treatment option.

Moreover, the aim of chemotherapy for advanced cancer is to seek a cure and at least improve survival time with good QOL. Non-hematological toxicities substantially worsen patient QOL and lead to poor survival outcomes. The current study showed that a low incidence of severe nonhematological toxicities, including grade 3 peripheral sensory neuropathy, might enhance OS while maintaining QOL. Nab-paclitaxel allows safe infusion with shorter infusion schedules as compared to solvent-based paclitaxel, with no requirement of premedication against solvent-based hypersensitivity reactions. In addition, low dose tri-weekly administration of nab-paclitaxel is convenient in enabling less frequent outpatient visits.

Recently, a phase II study of nab-paclitaxel in combination with ramucirumab showed promising activity against previously treated advanced gastric cancer patients. ORR was $54.8 \%$ and DCR was $92.9 \%$ (22). Though grade 3 peripheral sensory neuropathy was not observed, grade $3 / 4$ neutropenia occurred in $76.7 \%$ of patients, which was more frequent than that observed in the weekly nabpaclitaxel treatment in the ABSOLUTE trial (3) and in our study. For safety during chemotherapy, it may be difficult to apply nab-paclitaxel in combination with ramucirumab for patients with poor performance status or with a severe comorbidity.

This study has some notable limitations. First, this study was a single-arm phase II study in Japanese patients with a limited sample size. Second, we were unable to recruit the number of patients needed to reach the estimated sample size requirements. Third, we did not examine therapeutic outcomes using another reduced dose of triweekly nab-paclitaxel, such as by administering $80 \%$ of the regular dose. Therefore, it is advisable to conduct a randomized controlled trial (RCT) comparing the outcomes between the normal $\left(260 \mathrm{mg} / \mathrm{m}^{2}\right)$ and low dose of triweekly of nab-paclitaxel therapy as the second-line treatment for advanced gastric cancer in a sufficient number of patients. 
In conclusion, the tri-weekly low dose of nab-paclitaxel therapy is tolerable and has an acceptable safety. This regimen may be beneficial for selected patients who cannot use solvent-based paclitaxel and ramucirumab. The use of RCT to confirm the feasibility of this regimen is strongly suggested.

\section{Conflicts of Interest}

None of the Authors have any conflicts of interest to disclose regarding this study.

\section{Acknowledgements}

The Authors are grateful to Ms. Takako Ishibashi for her assistance in the study (YCU Center for Novel and Exploratory Clinical Trials). This was an investigator-initiated clinical trial that was not supported by any industry funding, investigated by the Yokohama Clinical Oncology Group (YCOG) and numbered YCOG 1405. YCOG is a research group investigating various concerns regarding surgery and oncology, composed of the Department of Gastroenterological Surgery, Yokohama City University Graduate School of Medicine, and its affiliated institutions. Five institutions of YCOG participants agreed on the contents of this prospective multicenter study and the protocol was approved by the institutional review board of each participating institutions.

\section{References}

12010 G Estimated Cancer Incidence, Mortality and Prevalence Worldwide in 2012: WHO. International Agency for Research on Cancer. http://globocan.iarc.fr/Pages/fact_sheets_cancer.aspx.

2 Japan CRaSi (2014): National Cancer Center. Center for Cancer Control and Information Services. http://ganjoho.jp/reg_stat/ statistics/stat/summary.html.

3 Shitara K, Takashima A, Fujitani K, Koeda K, Hara H, Nakayama N, Hironaka S, Nishikawa K, Makari Y, Amagai K, Ueda S, Yoshida K, Shimodaira H, Nishina T, Tsuda M, Kurokawa Y, Tamura T, Sasaki Y, Morita S and Koizumi W: Nab-paclitaxel versus solvent-based paclitaxel in patients with previously treated advanced gastric cancer (ABSOLUTE): an open-label, randomised, non-inferiority, phase 3 trial. Lancet Gastroenterol Hepatol 2(4): 277-287, 2017.

4 Ford HE, Marshall A, Bridgewater JA, Janowitz T, Coxon FY, Wadsley J, Mansoor W, Fyfe D, Madhusudan S, Middleton GW, Swinson D, Falk S, Chau I, Cunningham D, Kareclas P, Cook N, Blazeby JM and Dunn JA: Docetaxel versus active symptom control for refractory oesophagogastric adenocarcinoma (COUGAR-02): an open-label, phase 3 randomised controlled trial. Lancet Oncol 15(1): 78-86, 2014.

5 Kang JH, Lee SI, Lim DH, Park KW, Oh SY, Kwon HC, Hwang IG, Lee SC, Nam E, Shin DB, Lee J, Park JO, Park YS, Lim HY, Kang WK and Park SH: Salvage chemotherapy for pretreated gastric cancer: a randomized phase III trial comparing chemotherapy plus best supportive care with best supportive care alone. J Clin Oncol 30(13): 1513-1518, 2012.

6 Thuss-Patience PC, Kretzschmar A, Bichev D, Deist T, Hinke A, Breithaupt K, Dogan Y, Gebauer B, Schumacher G and Reichardt
P: Survival advantage for irinotecan versus best supportive care as second-line chemotherapy in gastric cancer - a randomised phase III study of the Arbeitsgemeinschaft Internistische Onkologie (AIO). Eur J Cancer 47(15): 2306-2314, 2011.

7 Dorr RT: Pharmacology and toxicology of Cremophor EL diluent. Ann Pharmacother 28: S11-14, 1994.

8 Gelderblom $\mathrm{H}$, Verweij $\mathrm{J}$, Nooter $\mathrm{K}$ and Sparreboom A: Cremophor EL: the drawbacks and advantages of vehicle selection for drug formulation. Eur J Cancer 37(13): 1590-1598, 2001.

9 Ibrahim NK, Desai N, Legha S, Soon-Shiong P, Theriault RL, Rivera E, Esmaeli B, Ring SE, Bedikian A, Hortobagyi GN and Ellerhorst JA: Phase I and pharmacokinetic study of ABI-007, a Cremophor-free, protein-stabilized, nanoparticle formulation of paclitaxel. Clin Cancer Res 8(5): 1038-1044, 2002.

10 Nyman DW, Campbell KJ, Hersh E, Long K, Richardson K, Trieu V, Desai N, Hawkins MJ and Von Hoff DD: Phase I and pharmacokinetics trial of ABI-007, a novel nanoparticle formulation of paclitaxel in patients with advanced nonhematologic malignancies. J Clin Oncol 23(31): 7785-7793, 2005.

11 Desai N, Trieu V, Yao Z, Louie L, Ci S, Yang A, Tao C, De T, Beals B, Dykes D, Noker P, Yao R, Labao E, Hawkins M and Soon-Shiong P: Increased antitumor activity, intratumor paclitaxel concentrations, and endothelial cell transport of cremophor-free, albumin-bound paclitaxel, ABI-007, compared with cremophor-based paclitaxel. Clin Cancer Res 12(4): 13171324, 2006.

12 Gardner ER, Dahut WL, Scripture CD, Jones J, Aragon-Ching JB, Desai N, Hawkins MJ, Sparreboom A and Figg WD: Randomized crossover pharmacokinetic study of solvent-based paclitaxel and nab-paclitaxel. Clin Cancer Res 14(13): 42004205, 2008.

13 Sasaki Y, Nishina T, Yasui H, Goto M, Muro K, Tsuji A, Koizumi W, Toh Y, Hara T and Miyata Y: Phase II trial of nanoparticle albumin-bound paclitaxel as second-line chemotherapy for unresectable or recurrent gastric cancer. Cancer Science 105(7): 812-817, 2014.

14 Ibrahim NK, Samuels B, Page R, Doval D, Patel KM, Rao SC, Nair MK, Bhar P, Desai N and Hortobagyi GN: Multicenter phase II trial of ABI-007, an albumin-bound paclitaxel, in women with metastatic breast cancer. J Clin Oncol 23(25): 60196026, 2005.

15 Yamamoto S, Maeda N, Nagashima Y, Kubo H, Sato Y, Matsui H, Inoue Y, Shindo Y, Kanekiyo S, Sakamoto K, Suzuki N, Takeda S, Ueno T, Yoshino S, Hazama S, Oka M and Nagano $\mathrm{H}$ : A phase II, multicenter, single-arm study of tri-weekly lowdose nanoparticle albumin-bound paclitaxel chemotherapy for patients with metastatic or recurrent breast cancer. Breast Cancer 24(6): 783-789, 2017.

16 Common Terminology Criteria for Adverse Events v4.0: Institute NC. https://evs.nci.nih.gov/ftp1/CTCAE/About.html.

17 Eisenhauer EA, Therasse P, Bogaerts J, Schwartz LH, Sargent D, Ford R, Dancey J, Arbuck S, Gwyther S, Mooney M, Rubinstein L, Shankar L, Dodd L, Kaplan R, Lacombe D and Verweij J: New response evaluation criteria in solid tumours: revised RECIST guideline (version 1.1). Eur J Cancer 45(2): 228-247, 2009.

18 Wilke H, Muro K, Van Cutsem E, Oh SC, Bodoky G, Shimada Y, Hironaka S, Sugimoto N, Lipatov O, Kim TY, Cunningham D, 
Rougier P, Komatsu Y, Ajani J, Emig M, Carlesi R, Ferry D, Chandrawansa K, Schwartz JD and Ohtsu A: Ramucirumab plus paclitaxel versus placebo plus paclitaxel in patients with previously treated advanced gastric or gastro-oesophageal junction adenocarcinoma (RAINBOW): a double-blind, randomised phase 3 trial. Lancet Oncol 15(11): 1224-1235, 2014.

19 Association JGC (2018): Japanese gastric cancer treatment guidelines 2018 (ver. 5).

20 Goedde HW, Agarwal DP, Fritze G, Meier-Tackmann D, Singh S, Beckmann G, Bhatia K, Chen LZ, Fang B, Lisker R, Paik YK, Rothhammer F, Saha N, Segal B, Srivastava LM and Czeizel A: Distribution of ADH2 and ALDH2 genotypes in different populations. Hum Genet 88(3): 344-346, 1992.

21 Riondino S, Del Monte G, Fratangeli F, Guadagni F, Roselli M and Ferroni P: Anti-angiogenic drugs, vascular toxicity and thromboembolism in solid cancer. Cardiovasc Hematol Agents Med Chem 15(1): 3-16, 2017.
22 Bando H, Shimodaira H, Fujitani K, Takashima A, Yamaguchi K, Nakayama N, Takahashi T, Oki E, Azuma M, Nishina T, Hironaka S, Komatsu Y and Shitara K: A phase II study of nabpaclitaxel in combination with ramucirumab in patients with previously treated advanced gastric cancer. Eur J Cancer 91: 8691, 2018.
Received October 25, 2018

Revised November 5, 2018

Accepted November 6, 2018 